\title{
EL FENÓMENO DEL SOFT LAW Y LAS NUEVAS PERSPECTIVAS DEL DERECHO INTERNACIONAL*
}

\author{
Mauricio Iván del TORO HUERTA**
}

RESUMEN: El derecho internacional es un sistema en constante transformación. En los últimos años nuevos procesos de creación normativa han motivado el replanteamiento de la idea tradicional de las fuentes del derecho internacional. Es en este contexto donde se desarrolla la problemática del denominado soft law internacional. Así, la expresión soft law ha sido duramente criticada por su ambigüedad y redundancia por algunos, y exaltada y promovida por otros. No existe un concepto universalmente aceptado y su contenido es difuso. Sin embargo, el soft law parece haber ganado terreno dentro de la discusión académica. En el fondo, la reflexión sobre el soft law abre la puerta hacia una concepción mucho más abierta, compleja y plural del derecho internacional, acorde con los tiempos de mayor globalización mundial. Ello no está ausente de riesgos frente a los nuevos fenómenos de unilateralismo estatal. Este es el tema del presente trabajo.

ABSTRACT: International law is a normative system in constant transformation. Over the last years new normative creation processes have motivated the reassessment of the traditional idea of the sources of international law. It is in this context where the problem of the international soft law takes place. The concept of soft law has been criticized by some because of its ambiguity and redundancy, and exalted and promoted by others. There is not a universally accepted concept of soft law and its content is diffuse. Notwithstanding, soft law seems to have won its own place in the academic discussion. At the bottom, the reflection on soft law opens the door toward a much more open, complex and plural conception of international law, in relation with the times of world globalization. It's not risk free in an era of new states' unilateralisms. This is the topic of the present paper.

RÉSUMÉ: Le droit internationale est un système normatif en constante transformation. Au cours des dernières années, de nouveaux processus de création normative a motivé la réévaluation de l'idée traditionnelle des sources du droit internationale. C'est dans ce contexte où le débat sur la soft law internationale repose. La notion de soft law a été critiqué par les uns pour son ambiguité et sa redondance, et exalté et encouragé par les autres. Il n'existe toujours pas de concept universellement accepté de soft law et son conte$n u$ est diffus. Néanmoins, la soft law paraît avoir fait sa propre place dans la discussion doctrinale. Au fond, la réflexion sur soft law ouvre la porte vers une conception beaucoup plus ouverte, complexe et plurielle du droit international, en accord avec les temps de globalisation de monde. Ce n'est pas sans risques surtout devant les nouveaux phénomènes d'unilateralismes de quelques états. La soft law est le sujet de ce texte.

* El autor desea agradecer el apoyo del Consejo Nacional de Ciencia y Tecnología en la elaboración de este trabajo, desarrollado en el marco de las actividades de investigación de la beca que le ha sido otorgada por dicha institución para la realización de estudios de postgrado.

** Profesor de derecho internacional público. Facultad de Derecho, UNAM. 
SUMARIO: I. A manera de introducción. II. El origen de la discusión: el derecho internacional como modelo dicotómico o normatividad relativa. III. Breve referencia a la teoría de las fuentes del derecho internacional. IV. La teoría de las fuentes y el fenómeno del soft law. V. Las formas del soft law. VI. Características generales de los instrumentos del soft law. VII. Las razones del soft law y sus consecuencias. VIII. Comentario final: el soft law en tiempos de unilateralismos. IX. Bibliografia.

\section{A MANERA DE INTRODUCCIÓN}

El derecho internacional, como todo ordenamiento jurídico, es un sistema en constante transformación. En su desarrollo, y en la medida en que trata de adaptarse a la dinámica de los distintos actores de la sociedad internacional, se han incorporado, tanto en su estructura teórica como institucional, nuevos elementos de análisis. Cada vez más, nuevas situaciones y fenómenos que se presentan en la comunidad internacional reclaman para sí un tratamiento jurídico. Piénsese, por ejemplo, en la creciente internacionalización de los derechos humanos y su repercusión en el ámbito tradicional de la soberanía y el dominio reservado; en las denominadas intervenciones humanitarias; en la creación de instituciones supranacionales políticas o judiciales, como la Corte Penal Internacional o el proceso de unificación europeo; en el derecho al desarrollo; en la protección del medio ambiente, la cuestión del cambio climático y la protección a la capa de ozono; en el avance biotecnológico y los recursos genómicos, en la creciente crisis por suministros de agua potable; en el avance en la comunicación y la internet, etc. ${ }^{1}$

A lo largo del siglo XX, los cambios fundamentales en la disciplina sobrevinieron con la reestructuración de la sociedad internacional a partir

1 Sobre algunos de estos temas véase: Brena Sesma, Ingrid, Díaz Müller, Luis T., Segundas Jornadas sobre globalización y derechos humanos: bioética y biotecnología, UNAM, México, 2004; Giles Carnero, Rosa, La amenaza contra la capa de ozono y el cambio climático: respuesta jurídico-internacional, Universidad de Huelva, España, 2003; García Ramírez, Sergio, La Corte Penal Internacional, 2a ed., México, Inacipe, 2004; Martín, Claudia, Rodríguez-Pinzón, Diego y Guevara, José, Derecho internacional de los derechos humanos, México, Fontamara, 2004. Sobre algunas de las trasformaciones del derecho internacional en nuestros días véase: Lagos, Enrique, "Algunas tendencias del derecho internacional a principios del siglo XXI", Anuario Mexicano de Derecho Internacional, vol. V, 2005, pp. 309-335. 
de la segunda posguerra y no han dejado de producirse hasta nuestros días impulsados por fenómenos complejos como la globalización, el fin del mundo bipolar o el surgimiento de nuevos unilateralismos. En un primer momento dichos cambios se manifestaron, entre otros aspectos, en la fundación de la Organización de las Naciones Unidas y otras organizaciones de carácter regional, así como en el acelerado incremento del número de organismos y agencias internacionales de cooperación; en el proceso de descolonización y autodeterminación de los pueblos y el consiguiente aumento en el número de Estados independientes en la sociedad internacional con representación en la Asamblea General de las Naciones Unidas. Posteriormente, otros fenómenos impactaron el sistema internacional: la reestructuración mundial producida al término de la guerra fría y el surgimiento de nuevos nacionalismos; el impacto del proceso de globalización mundial, tanto es su aspecto económico y financiero como en su aspecto científico y humanitario; la importancia creciente de actores no gubernamentales en la escena internacional, en particular las empresas transnacionales y las organizaciones no gubernamentales, pero también, el impacto criminal de mafias transnacionales como el narcotráfico y el terrorismo. Todo ello hace que el derecho internacional, no obstante conservar sus principios fundamentales, haya tenido que incorporar en su dinámica a nuevos actores y sujetos internacionales y reconocer nuevas situaciones, lo mismo que ampliar su campo de aplicación hacia otros ámbitos antes considerados asuntos domésticos o bien hacia espacios comunes, como los fondos marinos y oceánicos, la antártica, el espacio ultraterrestre, la luna, etc.

La idea tradicional de las fuentes del derecho internacional no ha sido la excepción y nuevos procesos de creación normativa reclaman su reconocimiento. Como ha advertido Julio Barberis, el "orden internacional actual no constituye un sistema cerrado en el que existe un número determinado y limitado de modos de creación de normas jurídicas. Los miembros de la comunidad internacional pueden acordar nuevas fórmulas para crear el derecho de gentes". ${ }^{2}$ En consecuencia el derecho internacional está abierto a la transformación, reflejo evidente de la cambiante y compleja sociedad que regula o pretende regular: Ubi societas, ibi jus.

2 Cfr. Barberis, Julio, Formación de derecho internacional, Buenos Aires, Ábaco Rodolfo de Palma, 1994, p. 257. 
Es en este contexto donde se desarrolla la problemática del denominado soft law o weak law, ${ }^{3}$ o como se le ha denominado en castellano: "derecho flexible", "pre-derecho", "derecho blando o en agraz". ${ }^{4}$ Pues, normalmente la discusión en torno al concepto de soft law se sitúa en el amplio contexto de la reflexión permanente sobre las "fuentes" del derecho internacional público. ${ }^{5}$ Así, la expresión soft law parece haber ganado terreno (a pesar de las fuertes criticas que ha recibido) ${ }^{6}$ y se presenta dentro de la doctrina como una pieza que si bien no acaba de encajar todavía en el amplio rompecabezas del derecho internacional contemporáneo sí tiene ya ganado su lugar en el debate académico sobre los problemas en la formación de obligaciones internacionales. ${ }^{7}$

El presente trabajo se sitúa dentro de la problemática relativa a la naturaleza del denominado soft law y a su influencia en el ámbito de la formación del derecho internacional. Sin pretender resolver aquí la cuestión, a continuación se propone un marco general que permita situar la discusión en un plano de entendimiento de un fenómeno innegable cuya importancia ha sido y seguirá siendo motivo de reflexión. El planteamiento del tema, sin embargo, evita situar la discusión en el ámbito tradi-

3 Para una revisión global del tema véase: Shelton, Dinah (ed.), Commitment and compliance. The role of non-binding norms in the international legal system, Oxford University Press, New York, 2000. Sobre los aspectos generales del concepto véase, entre otros: Thürer Daniel, "Soft Law", en R. Bernhardt (ed.), Encyclopedia of Public International Law, 2000, pp. 454-460, Baxter, R. R., "International law in "her infinite variety", International and Comparative Law Quarterly, Vol. 29, October, 1980, pp. 549-566.

4 Cfr. Gutiérrez Espada, Cesáreo, Derecho internacional público, Editorial Trotta, Madrid, 1995, pp. 617-618; Remiro Brotóns, Antonio; Riquelme, Rosa; Orihuela, Esperanza; Díez-Hochleitner, Javier y Pérez-Prat, Luis, Derecho internacional, McGraw Hill, Madrid, 1997, p. 1088; Pastor Ridruejo, José A., Curso de derecho internacional público y organizaciones internacionales, 8a. ed., Tecnos, Madrid, 2002, pp. 156-157. Otros autores han hablado de la diplomacia parlamentaria por ejemplo: Sepúlveda, César, "La diplomacia parlamentaria y la formación del nuevo orden jurídico internacional", en Estudios en homenaje al profesor Miaja de la Muela, Tomo II, Tecnos, Madrid, 1979, pp. 773-791.

5 Cfr. Entre otros: Hoof, GJH van, Rethinking the Sources of International Law, Kluwer Law, The Netherlands, 1983, pp. 179 y ss; Wellens, K.C y Borchardt, G.M., "Soft Law in European Community Law", European Law Review, October, 1989, p. 267; Shelton. Dinah, "International Law add 'relative normativity" en Evans, Malcolm (ed.), International Law, Oxford, University Press, 2003.

6 Cfr. Klabbers, Jan, "The redundancy of soft law", Nordic Journal of International Law 65, 1996, pp. 167-182.

7 Sobre el tema véase el debate suscitado en la 82a. reunión anual de la American Society of International Law entre los profesores Handl, Reisman, Simma, Dupuy y Chinkin en: "A Hard Look at Soft law" en American Society of International Law Proceedings, $82 \mathrm{Am}$. Soc'y Int'l L. Proc. (1988), pp. 371-395. 
cional de las denominadas "fuentes" del derecho internacional, ya que tal enfoque se presta a confusiones y ambigüedades, pues incluso el concepto mismo de "fuentes" esta siendo revisado por la doctrina en la actualidad (aunque mantenga su sentido y valor pragmático y académico). Asimismo, no se considera que la discusión sobre la naturaleza del fenómeno deba plantearse exclusivamente desde la perspectiva de la dicotomía soft law/hard law, binding/non-binding, derecho-duro/derecho-blando u obligatorio/no-obligatorio, si por ello atendemos solamente aspectos formales en la creación de las normas ${ }^{8}$ (aunque existen buenas razones y fuertes argumentos para adherirse a tal enfoque que, entre otras cosas, desenmascara posibles motivaciones ideológicas ocultas tras ciertos discursos retóricos sobre las bondades del soft law en el marco del relativismo y el unilateralismo estatal). Tal perspectiva dicotómica creemos descarta, sin más, una reflexión profunda sobre la propia naturaleza del derecho internacional y sobre la estructura operativa del discurso jurídico, cuya aportación más significativa consiste, en el fondo, en proponer un cambio de paradigma en la concepción general del orden jurídico internacional que se adapte a las nuevas dinámicas de la comunidad internacional en un mundo cada vez más interdependiente y globalizado.

Estimo, por el contrario, que la inclusión del debate en torno al soft law debe hacerse desde una perspectiva global del derecho internacional pues, entre otras cosas, enriquece la concepción de los procesos de formación de derechos y obligaciones internacionales; pone de manifiesto el papel de los diferentes actores de la sociedad internacional y sus estrategias de acción; así como aporta destellos sobre la posible mecánica del derecho internacional en los años venideros. Si el término es apropiado o conveniente, si carece de certezas o abunda en imprecisiones, si encuentra un referente en la práctica nacional o internacional, o si amenaza el futuro del derecho internacional o lo complementa, serán cuestiones que seguirán en el debate y que aquí, por supuesto, no pretenden resolverse.

A continuación, después de presentar el origen de la discusión, se hace una breve referencia a la teoría de las fuentes del derecho internacional como punto de partida para trazar posteriormente un esbozo del

$8 \mathrm{Al}$ respecto, véase: Zemanek, Karl, "Is the term 'Soft law' convenient?", en Liber Amicorum Professor Seidl-Hohenveldern, Kluwer, 1998, pp. 845; Abbott, Kenneth W. y Snidal Duncan, "Hard and soft law in international governance", International Organization, vol. 54, 2000, p. 424 . 
concepto de soft law, con el objeto de identificar sus principales características y efectos para, finalmente, exponer las razones por las cuales se considera que la teoría del soft law (si se le puede llamar así) presenta más que una descripción neutral de un fenómeno, un nuevo modelo de pensar al derecho internacional no exento también de ambigüedades y, por supuesto, sumergido en las aguas pantanosas de la política internacional en una época compleja y propensa a lo que Antonio Remiro Brotóns llama, la "desvertebración del derecho internacional público en la sociedad globalizada". ${ }^{9}$

\section{EL ORIGEN DE LA DISCUSIÓN. EL DERECHO INTERNACIONAL COMO MODELO DICOTÓMICO O NORMATIVIDAD RELATIVA}

Aunque se reconoce que el término soft law fue introducido al lenguaje del derecho internacional por Lord McNair, ${ }^{10}$ lo cierto es que éste acuñó el término para distinguir entre proposiciones de lege lata y de lege ferend $a^{11}$ y no para distinguir un fenómeno complejo que supone la existencia de variaciones normativas que van desde lo no vinculante hasta lo vinculante, del "no derecho" al derecho, de lo soft a lo hard. Con la expresión soft law se trataba de describir enunciados formulados como principios abstractos, presentes en todo ordenamiento jurídico, que devenían operativos a través de su aplicación judicial. ${ }^{12}$ El lugar del soft law

9 Cfr. Remiro Brotóns, Antonio, "Desvertebración del Derecho Internacional en la sociedad globalizada" en Cursos Euromediterráneos Bancaja de Derecho Internacional, vol. V, Tirant lo blanch, Valencia, 2001, pp. 46-381. Para una explicación del fenómeno del soft law desde la perspectiva de la globalización véase: O’Connell, Mary Ellen, “The role of Soft Law in a Global Order", en Shelton, Dinah (ed.), Commitment and Compliance..., cit, pp. 100-114.

$10 C f r$. Dupuy, R. J., "Declaratory law and programmatory law: from revolutionary custom to 'soft law”, en R.J. Akkerman, P.J. van Krieken \& Ch. O. Pannenborg (eds.), Declarations on principles, a quest for world peace (Liber Röling), Sijthoff, Leyden, 1997, 247-257; Casanovas y La Rosa, Oriol, "Unidad y pluralismo en derecho internacional público" en Curso Euromediterraneo Bancaja de Derecho Internacional, vol. II, 1998, pp. 114 y ss; Hillgenberg, Hartmut, "A Fresh Look at Soft Law", European Journal of International Law, núm.. 3, 1999, pp. 499-515; Thürer Daniel, "Soft Law", en R. Bernhardt (ed.), Encyclopedia of Public International Law, 2000, pp. 454-460.

11 Cfr. Jennings, R.Y., "An International Lawyer takes Stock”, International \& Comparative Law Quarterly, 1990, vol. 39, pp. 513-529.

12 Cfr. Mazuelos Bellido, Ángeles, "Soft Law: ¿Mucho ruido y pocas nueces", Revista Electrónica de Estudios Internacionales, 2004, disponible en www.reei.org. 
en el debate académico como se manifiesta en nuestros días vendría a configurarse después.

Con el paso del tiempo y el correr de la tinta muchos otros autores adoptaron tal expresión y la dotaron de significado o al menos de cierto contenido. ${ }^{13}$ Así, la expresión soft law busca describir la existencia de fenómenos jurídicos caracterizados por carecer de fuerza vinculante aunque no carentes de efectos jurídicos o al menos con cierta relevancia jurídica. Ello supone la existencia de una normatividad relativa en el sistema internacional y la consecuente afrenta al modelo dicotómico tradicional.

En este sentido, es conocido el debate iniciado a raíz de la publicación de algunos trabajos del profesor Prosper Weil sobre los efectos de estos fenómenos en la formación de una normatividad relativa y sus riesgos para el derecho internacional. ${ }^{14}$ En su polémico artículo "Towards Relative Normativity in Internacional Law?" el autor analiza los posibles riesgos de algunos desarrollos recientes (para finales de la década de los setenta y comienzos de los años ochenta del siglo XX) en el ámbito del derecho internacional (por ejemplo, la supuesta normatividad relativa, la teoría del jus cogens, la distinción entre delitos y crímenes internacionales adoptada en ese entonces en los trabajos de la Comisión de Derecho Internacional en su proyecto de artículos sobre responsabilidad internacional, la noción de obligaciones erga omnes, etc.) que si bien en sí mismos se presentaban como benéficos podrían convertirse en posibles patologías del sistema internacional. En particular, Weil considera que una excesiva variación en la normatividad internacional supondría el desconocimiento de la naturaleza del propio ordenamiento internacional, como derecho de cooperación entre entidades soberanas, y de sus procesos de formación normativa identificados principalmente con el voluntarismo estatal.

Sobre el pretendido valor de las resoluciones de organismos internacionales, instrumentos jurídicos asociados tradicionalmente con el término soft law, Weil considera que no constituyen "fuentes" formales del

13 Cfr. Dupuy, R. J. "Declaratory law and programmatory law: from revolutionary custom to 'soft law", op. cit.; Seidl-Hohenveldern, Ignaz, "International Economic 'Soft Law", 163 Recueil des Cours (1979, t. II) pp. 173-246; Baxter, R. R., "International law in "her infinite variety", International and Comparative Law Quarterly, vol. 29, October, 1980, pp. 549-566.

14 Weil, Prosper, "Towards Relative Normativity in Internacional Law?", 77 American Journal of Internacional Law, 1983, pp. 413-442. Éste artículo es la traducción y ampliación de un trabajo anterior publicado en 89 Rev. Générale Droit Int'l Public 5 (1982). 
derecho internacional, aunque no por ello deban ser ignoradas por los juristas internacionales. En opinión del profesor Weil esto no significa que exista un sistema escalonado de normas internacionales, por tanto las resoluciones de organizaciones internacionales no suponen la existencia de una nueva categoría de normas, sino la constatación de un proceso de producción normativa basado en la distinción entre lex lata y lege feren$d a$. De tal forma que la acumulación de consideraciones de lege ferenda, esto es de "nonlaw" o "prelaw", no es suficiente para crear derecho. ${ }^{15}$

Para este autor, si bien es cierto que a la distinción entre soft law y hard law pudiera atribuirse algunos "beneficios", tales como, estimular una mayor "conciencia jurídica", contribuir a asegurar la presencia de valores éticos en el proceso de positivización del derecho internacional o reflejar la diversidad de la comunidad internacional; lo cierto es que —en opinión del profesor Weil- esta supuesta gradación puede convertirse en un fenómeno patológico que amenace la naturaleza positiva del derecho internacional. El hecho de que muchos autores no reconozcan la diferencia entre normas jurídicas y "no-normas" no necesariamente incide en la práctica de los Estados en tanto que éstos continúan percibiendo claramente tal diferencia. Desde esta perspectiva, la relativización de la normativa internacional puede derivar también en la relativización de los derechos y obligaciones internacionales, lo que supone un evidente riesgo de minimizar las obligaciones internacionales y difuminar los sujetos obligados. ${ }^{16}$ Por ello es preciso un adecuado control de tales fenómenos de relativización. En síntesis Weil considera:

Accordingly, the potential negative consequences of the relativization of international normativity must at worst be regarded as secondary effects of changes that in themselves are beneficial. Vigilance, however, is imperative, lest too high a price be paid for the progress of international law towards greater moral substance and greater solidarity. At a time when international society needs more than ever a normative order capable of ensuring the peaceful coexistence, and cooperation in diversity, of equal and equally sovereign entities, the waning of voluntarism in favor of the ascendancy of some, neutrality in favor of ideology, positivity in favor of 
ill-defined values might well destabilize the whole international normative system and turn into an instrument that can no longer serve its purpose. ${ }^{17}$

El artículo del profesor Weil suscitó respuestas inmediatas. ${ }^{18}$ Por mencionar sólo algunos ejemplos, respecto del posible efecto patológico del relativismo normativo, el profesor Ulrich Fastenrath considera que en todo sistema legal existe una gradación normativa y que los conceptos y enunciados jurídicos sólo otorgan una aparente certeza en el sistema jurídico que en todo caso están sujetos a interpretación. En un mundo heterogéneo y plural, el fenómeno del soft law puede contribuir a esclarecer muchos de los consensos que se expresan en normas de hard law. En palabras del profesor Fastenrath:

Gradation in the normativity of the law has proven to be unavoidable in all legal theories. Legal propositions (Rechtssätze) and the legal concepts employed in them only give an appearance of certainty. With the extension of international law beyond the boundaries of the fairly homogeneous Western State system, uncertainty has even increased. The use of language and the intellectual background of comprehension, which form the basis for the interpretation of legal norms, are never identical. Rather, with increasing cultural diversity, they will deviate more and more from one another. The more heterogeneous the world becomes, the more importance must be attached to the formulation of common goals and criteria for the balancing of interests and to the establishment of all-encompassing linguistic conventions. In the face of conflicting interests and divergent goals, stemming from different cultures, clarity in the formulation and comprehension of legal propositions (Rechtssätze) can only be preserved in this manner, na-

17 Ibidem, p. 423.

18 Entre otras: Fastenrath, Ulrich, "Relative Normativity in International Law", 4 European Journal of International Law, 1993, pp. 305-340; Tasioulas, John, "In Defence of Relative Normativity. Communitarian Values and the Nicaragua Case" en Oxford Journal of Legal Studies, vol. 16, núm. 1, 1996, pp. 85-128. En este artículo, Tasioulas cita la opinion de Richard Falk como unas de las más críticas y escépticas de la posición de Weil, al señalar: "I am not so impressed by Prosper Weil's article. In fact I would welcome it if someone could enlighten me as to why it is considered such an important formulation. It strikes me as being, to put it rather strongly, a slightly hysterical effort to stop the world from changing. I see it as the work of a highly intelligent person who dislikes what is happening in the world and is translating into jurisprudence a kind of ideological nostalgia for an earlier period of certainty and consensus; he dislikes the problems of tension and diversity that exist in the world. I would stress that I am putting this so impolitely because I would really like to understand why others find the article so important and useful. In my opinion it is a gross overstatement of what is happening and makes use of extremely emotionally loaded language in order to express a highly nostalgic view of international political life". 
rrowing the room for possible interpretations and thus ensuring that the behaviour of all actors may remain predictable. Here, the much-maligned phenomenon of soft law performs an invaluable function. It enables worldwide agreement on the content of hard law, in that it limits the scope of acceptable subjective auto-determination. Thus, soft law constitutes a 'positivized' text, even though an extra-legal one in the eyes of legal positivism, which saves this very theory from extinction in a pluralist world. The fact that this function has been largely ignored is frequently due to the assumption that one's own method of legal comprehension is the only correct one. ${ }^{19}$

Como es fácil de observar con esta muestra, la polémica sobre el soft law está lejos de ser resuelta. Para algunos autores, como Jan Klabbers, no existe una constatación en la práctica internacional o nacional de la existencia autónoma de dicho fenómeno; además, la dicotomía obligatorio/no-obligatorio, derecho/no-derecho, etc., subsume perfectamente la idea de soft law en tanto que en todo orden jurídico existen algunas normas más importantes que otras, más o menos precisas, pero nunca más o menos obligatorias, por tanto la expresión soft law resulta ambigua y redundante. En palabras del autor:

Our binary law is well capable of handling all kinds of subtleties and sensitivities; within the binary mode, law can be more or less specific, more or less exact, more or less determinate, more or less wide in scope, more or less pressing, more or less serious, more or less far-reaching; the only thing it cannot be is more or less binding. ${ }^{20}$

Desde esta misma perspectiva dicotómica, Julio Barberis considera que son tres las principales acepciones del término soft law, a saber: a) las normas que se encuentran en proceso de formación y aún no han adquirido validez jurídica; $b$ ) las normas jurídicas de contenido difuso o vago en las que resulta difícil precisar si sus disposiciones han sido o no cumplidas debidamente; y c) las normas que se hallan en las resoluciones de la Asamblea General de las Naciones Unidas y de algunas organizaciones regionales, en los acuerdos políticos entre los gobiernos, en los "gentlemen's agreements", en ciertos códigos de conducta, en declaraciones conjuntas de presidentes o de ministros de Relaciones Exteriores, en di-

19 Fastenrath, Ulrich, "Relative Normativity in International Law", op. cit., p. 338.

20 Klabbers, Jan, "The redundancy of soft law", op. cit., p. 181. 
rectivas adoptadas por consenso en conferencias internacionales, etc., que en conjunto formarían un "orden jurídico intermedio".

Para el profesor Barberis el concepto de soft law en su primera acepción no es sino una injustificada forma de denominar a aquellas normas que se encuentran en proceso de consolidación y que la doctrina distingue ya con la referencias a la lex lata y lex ferenda; en el segundo caso la diferencia entre hard law y soft law no estaría dada por un distinto grado de obligatoriedad establecido por la norma jurídica sino por la dificultad (mayor o menor) de comprobar su incumplimiento, por lo que la expresión soft law no genera sino confusión pues en cualquiera de ambos casos las normas son obligatorias y las consecuencias jurídicas de su incumplimiento son las mismas. Finalmente — para este autor - no es conveniente hablar de la existencia de un "orden jurídico intermedio" basado en la existencia de normas de diferente clase o naturaleza, pues el concepto de validez de cualquier norma (sea jurídica o no) no está sujeto a graduación y si bien existen diferentes tipos de derechos y obligaciones esto no supone merma ni gradación en su validez; en este sentido, o es derecho o no lo es. De esta forma, la idea de un derecho "blando" no aparece como razonable, siendo el soft law un producto de intereses políticos que buscan otorgar obligatoriedad a ciertos actos y ciertas resoluciones para satisfacer sus propios intereses. ${ }^{21}$

Sin embargo, también es posible aproximarse al fenómeno del soft law desde una perspectiva diferente que concibe al derecho internacional $\mathrm{o}$, mejor dicho, a los efectos de las distintas normas e instrumentos internacionales en una escala que va de lo soft a lo hard y en consecuencia sin que sea necesario la exclusión de los opuestos como consecuencia del enfoque inevitable e irreducible de la dicotomía tradicional. Esta gradación del nivel de obligatoriedad responde tanto a conveniencias políticas como a la búsqueda de una mejor solución a determinada cuestión. Esta concepción parte del supuesto que en la sociedad internacional no todos los problemas pueden y deben encontrar una solución jurídica del tipo hard law, si no que, por el contrario, en ocasiones instrumentos no vinculantes ofrecen mejores soluciones, sea por que reflejan un mayor consenso de la comunidad internacional, por llenar alguna laguna, por esta- 
blecer criterios de interpretación o por ser la única vía de acuerdo posible. ${ }^{22}$

En opinión de W. Riphagen, dado que el derecho no es el único sistema normativo que rige la conducta humana, es preciso identificar los diferentes eslabones que llevan de lo no-jurídico a lo jurídico y para ello es preciso distinguir al menos cuatro escalones representados en la fórmula non-law/soft-law/law/ius cogens, en donde el soft law y el ius cogens representan los dos extremos del mundo jurídico y por tanto comparten cierta dificultad para su identificación. ${ }^{23}$ En el mismo sentido, Abbott y Snidal proponen analizar el soft law a partir de los diferentes grados de obligatoriedad, precisión y delegación de los textos, lo que supone una graduación continua del orden jurídico. En sus palabras, "it suggests the continuous gradations of hardness and softness that are blurred when the hard law/soft law distinction in incorrectly taken as binary". ${ }^{24}$

Desde esta perspectiva, la cuestión de la relatividad normativa o variable (relative normativity) está relacionada con la jerarquía de las normas en el derecho internacional y con la misma definición de derecho internacional, con su naturaleza, estructura y contenido. Por ello se afirma: "If and how the term 'soft law' should be used depends in large part on whether one adopts the binary or continuum view of international law". 25

Lo anterior muestra como el debate del soft law supone un debate más amplio entre concepciones del orden jurídico internacional. Por un lado, el modelo dicotómico derivado de la teoría tradicional de las "fuentes" del derecho internacional y, por el otro, el modelo teórico que supone la existencia de un sistema internacional escalonado y continuo en

22 Cfr. Chinkin, Christine, "Normative Development in the international legal system", en Shelton, Dinah (ed.), Commitment and compliance. The role of non-binding norms in the international legal system, op. cit., pp. 24 y ss; Abbott y Snidal consideran que "international actors choose softer forms of legalized governance when those form offer superior institutional solutions." Abbott y Snidal, "Hard and soft law in international governance", op. cit., p. 421.

23 Riphagen, W., "From soft law to ius cogens and back" en Victorian University of Wellington Law Review, vol. 17, núm. 2, April 1987, pp. 81-99.

24 Cfr. "Hard and soft law in international governance", op. cit., p. 424.

25 Shelton, Dinah, "International law and 'Relative Normativity",, en Evans, Malcolm (ed.), International Law, Oxford, University Press, 2003, p. 167. 
donde existe una normatividad relativa o variable. ${ }^{26}$ Ello nos lleva a considerar ahora el tema de las "fuentes" del derecho internacional.

\section{BREVE REFERENCIA A LA TEORÍA DE LAS FUENTES DEL DERECHO INTERNACIONAL}

El tema de las fuentes es uno de los ámbitos tradicionales de estudio y reflexión del derecho internacional y, como tal, su importancia es capital. Sin embargo, la noción de "fuentes del derecho internacional" en tanto heredada del léxico tradicional del derecho interno, en particular, de la clásica distinción entre fuentes formales, materiales e históricas, no esta exenta de ambigüedades e imprecisiones como reiteradamente lo ha destacado la doctrina. ${ }^{27}$

Generalmente el término "fuente" en derecho internacional se utiliza en un sentido amplio, esto es, en sus aspectos formales y materiales, ya que, como señala Ian Brownlie, ${ }^{28}$ el uso del término "fuente formal" puede resultar "inoportuno" y "engañoso" si se equipara a la terminología

26 Sobre las transformaciones en el orden internacional derivadas del reconocimiento del soft law y del ius cogens, el profesor Fastenrath señala: "The tendency inherent in soft law, to become a new form of law-making, cannot be denied. This observation is due to cause unrest in the orderly circles of classical international law, which is based on the sovereignty of States. However, a change of direction has already taken place both in and by means of international organizations. The tendency now is to view certain international issues as matters of concern to the international community as a whole... Another expression of this movement towards a global society may be seen in the recognition of some fundamental duties as constituting obligations erga omnes... Finally, the concept of ius cogens is another indication of an emerging global society. Through this instrument, worldwide perceptions of justice may be expressed. Whereas such perceptions have to date been crude and inconsistent, this merely points to the fact that we are but at the beginning of a process which has hitherto led us from the law of coexistence to the law of cooperation; the path to a true constitution of the global society remains open and untrodden before us. What we ought to do today is not to obstruct this path but to equip ourselves with the necessary instruments to tread it. In particular, we ought to incorporate soft law instruments into our existing legal methodology". Fastenrath, Ulrich, "Relative Normativity in International Law", cit., pp. 338-340.

27 Para una revisión general del tema de las fuentes desde una perspectiva crítica, véase: Sánchez Rodríguez, Luis Ignacio, "La apoteosis del consentimiento: de la noción de fuentes a los procesos de creación de derechos y de obligaciones internacionales", Anuario Hispano-Luso Americano de Derecho Internacional, vol. 16, 2003, pp. 191-234; Riedel, Eibe, "Standards and sources. Farewell to the exclusivity of sources triad in international law?", en European Journal of International Law, 1991, pp. 58-84; Abi-Saab, Geoges, "Les Sources du Droit International: Essai de Déconstruction" y Dupuy, Pierre-Marie, "Théorie des Sources et Coutume en Droit International Contemporain", en El derecho internacional en un mundo en transformación, Liber Amicorum en homenaje al profesor Eduardo Jiménez de Aréchaga, Uruguay, Fundación de Cultura Universitaria, 1994, t. 1, pp. 29-49 y 51-68.

28 Principles of Public International Law, 4a. ed, Oxford Clarendon Press, 1990, pp. 1-3. 
del derecho interno que lo identifica con el proceso legislativo, pues no existe semejante proceso a nivel internacional y, en cambio, una sentencia de la Corte Internacional de Justicia respecto de las partes en el proceso, una resolución adoptada por unanimidad de la Asamblea General de Naciones Unidas que evidencie la existencia de una práctica general obligatoria, o un tratado multilateral, a pesar de ser consecuencias de diferentes mecanismos de producción normativa, pueden tener los mismos efectos obligatorios en el plano internacional, lo mismo que en el ámbito interno una ley adoptada por el parlamento o una sentencia dictada por un tribunal constitucional.

En lugar de "fuentes formales", en sentido estricto, en derecho internacional existe el principio de que el consentimiento amplio de los Estados genera reglas de aplicación general. Esto es, una regla de derecho internacional es aquella que ha sido aceptada como tal por la comunidad internacional, ${ }^{29}$ siendo la costumbre y los tratados las fuentes principales del ordenamiento jurídico internacional, aunque no necesariamente las únicas formas de creación de derechos y obligaciones internacionales.

En este sentido, tradicionalmente se identifica al artículo 38 del Estatuto de la Corte Internacional de Justicia (CIJ) ${ }^{30}$ como la expresión básica de las distintas "fuentes" del derecho internacional. ${ }^{31}$ Sin embargo, se reconoce también ampliamente que la enumeración de las fuentes atribuida a este artículo, además de no establecer una jerarquía entre ellas,

29 Cfr. Henkin, Louis, Crawford Pugh, Richard; Shachter, Oscar; Smit, Hans, International law: cases and materials, 3a. ed., West Publishing co., St. Paul, Minn, 1993, p. 51.

30 "1. La Corte, cuya función es decir conforme al derecho internacional las controversias que le sean sometidas, deberá aplicar: a) las convenciones internacionales, sean generales o particulares, que establecen reglas expresamente reconocidas por los Estados litigantes; b) la costumbre internacional como prueba de una práctica generalmente aceptada como derecho; c) los principios generales de derecho reconocidos por las naciones civilizadas; d) las decisiones judiciales y las doctrinas de los publicistas de mayor competencia de las distintas naciones, como medio auxiliar para la determinación de las reglas de derecho, sin perjuicio de lo dispuesto por el artículo 59. 2. La presente disposición no restringe la facultad de la Corte para decidir un litigio 'ex aequo et bono', si las partes así lo convienen". El artículo 59 señala que "la decisión de la Corte no es obligatoria sino para las partes en litigio y respecto del caso que ha sido decidido".

31 En palabras de Brownlie: "Article 38 is generally regarded as a complete statement of the sources of international law”. (Op. cit., p. 3). Autores como José A. Pastor Ridruejo consideran que "para la determinación de las fuentes del Derecho Internacional Positivo es forzosa una referencia al apartado 1o. del artículo 38 del Estatuto." Posteriormente precisa que en su concepto las fuentes realmente autónomas son sólo dos: la costumbre y los tratados, aunque se reconozca también dentro de la expresión "fuentes del Derecho" a las situaciones individuales u obligaciones particulares (tratados bilaterales, las costumbres locales y los actos unilaterales). Pastor Ridruejo, José A., Curso de derecho internacional público, op. cit., p. 65. 
tampoco es exhaustiva.32 Incluso la propia noción de "fuentes" se considera ambivalente, equívoca e incompleta y se suele incluir dentro de las formas o procedimientos de creación de derechos y obligaciones internacionales ciertos actos unilaterales de los Estados, algunos comportamientos recíprocos, ${ }^{33}$ así como determinadas resoluciones de organizaciones internacionales. ${ }^{34}$ Por esas y otras razones, en la actualidad se atribuye al artículo 38 del Estatuto de la CIJ (redactado en el contexto de una época particular de la evolución del derecho internacional: 1920, como parte

32 De las diversas criticas que se hacen tanto a la identificación mecanicista de las "fuentes" del derecho internacional con el contenido del artículo 38 del Estatuto CIJ como de la propia noción de "fuentes" puede decirse, junto con Luis Ignacio Sánchez Rodríguez, respecto del primer aspecto que, si bien dicho artículo constituye "un precepto estimable por contener un catálogo descriptivo de las fuentes formales más habituales de nuestro ordenamiento y por su contenido mítico en la literatura y en la jurisprudencia internacionales... en modo alguno puede ser considerado: a) como un precepto convencional cuyo objeto y fin precisos radique en enunciar las fuentes del derecho internacional público; b) como un texto recoja todos los procedimientos posibles de creación de derechos y de obligaciones existentes en nuestro ordenamiento; c) como un mecanismo de ordenación jerárquica de las diferentes fuentes formales; d) como una referencia obligada, exclusiva y excluyente a la cual deba ajustarse la Corte, de manera castrense, para la solución judicial de las controversias internacionales, por no referirnos a otras jurisdicciones internacionales, como es el caso de la arbitral; e) como una fórmula de cristalización, inmutable e inmodificable, de todas las formas a través de las cuales se manifiesta el ordenamiento internacional." Respecto del segundo aspecto, la propia expresión de "fuentes" es ambivalente, pues enmascara la distinción entre fuentes formales y fuentes materiales, es equívoca ya que supone la existencia de un legislador universal y es incompleta al presuponer una simetría automática entre las normas y las obligaciones jurídicas e ignorar que, en determinados supuestos y bajo determinadas condiciones, las obligaciones internacionales pueden generarse al margen de las normas, como consecuencia del papel nuclear del consentimiento en el ordenamiento internacional. Cfr. Sánchez Rodríguez, Luis Ignacio, "La apoteosis del consentimiento: de la noción de fuentes a los procesos de creación de derechos y obligaciones internacionales", op. cit., pp. 193 y ss.

33 Cfr. Jiménez García, Francisco, Los comportamientos recíprocos en derecho internacional. A propósito de la aquiescencia, el estoppel y la confianza legítima, Editorial Dilex, España, 2002; Guerrero Peniche, Nicolás y Rodríguez Cedeño, Víctor, "Los actos unilaterales de los Estados en derecho internacional: los trabajos de codificación en la Comisión de Derecho Internacional", Anuario Mexicano de Derecho Internacional, vol. III, 2003, pp. 195-223; Barberis, Julio A., "Los actos jurídicos unilaterales como fuente del derecho internacional público, en Hacia un nuevo orden internacional y europeo. Estudios en homenaje al profesor don Manuel Díez de Velasco, Tecnos, Madrid, 1993, pp. 101-116; Virally, Michel, "Fuentes del derecho internacional", en Sorensen, Max (ed.), Manual de derecho internacional público, Fondo de Cultura Económica, México, 1973, pp. 149 y ss.

34 Sobre el valor jurídico de las resoluciones internacionales, véase, entre otros; Castañeda, Jorge, "Valor Jurídico de las Resoluciones de Naciones Unidas" en Obras Completas. I Naciones Unidas, El Colegio de México-SER, México, 1995, pp. 271-498; Tunkin, G. I., "The Role of Resolutions of International Organisations in Creating Norms of International Law" y Higgins, Rosalyn, "The Role of Resolutions of International Organizations in the Process of Creating Norms in the International System", ambos en Butler, W. E., International Law and The International System, Martinus Nijhoff Publishers, Dordrecht-Boston-Lancaster, 1987, pp. 5-20, y 21-30. 
del Estatuto de la Corte Permanente de Justicia Internacional, posteriormente incorporado al de la CIJ con leves modificaciones) un carácter procesal aunque no deja de considerarse una especie de "regla secundaria", utilizando el lenguaje de H. L. A. Hart. ${ }^{35}$ Asimismo, actualmente suele hablarse de procesos de formación de normas y creación de derechos y obligaciones internacionales (international lawmaking) más que de "fuentes" del derecho internacional, ${ }^{36}$ aunque dada su aceptación universal y su uso frecuente sigue siendo epígrafe y referencia común en muchos textos doctrinarios. ${ }^{37}$

\section{LA TEORÍA DE LAS FUENTES Y EL FENÓMENO DEL SOFT LAW}

Generalmente el ámbito del soft law se analiza en oposición al denominado hard law, entendiendo éste como aquellos instrumentos o prácticas generales con carácter obligatorio cuyo incumplimiento puede ser exigido por las vías institucionales de solución de conflictos y derivar en la responsabilidad internacional del Estado. Este modelo de aproximación al fenómeno desde la contraposición formal soft law/hard law busca identificar como derecho (hard) solamente aquellas normas que hayan sido producidas mediante las denominadas "fuentes" tradicionales del derecho internacional, en particular, mediante los tratados y la costumbre, dejando fuera del ámbito de lo jurídico otras manifestaciones de voluntad de los sujetos de derecho internacional.

Situar la discusión en torno al soft law en el ámbito de las "fuentes" del derecho internacional reconocidas en el artículo 38 del Estatuto de la CIJ implica suponer el carácter exhaustivo del mismo, en tanto que si dicho artículo establece las fuentes de producción de obligaciones internacionales es obvio que cualquier otro regla producida por algún mecanismo diverso queda fuera del denominado hard law. Sin embargo, tal aproximación parece incorrecta para explicar el fenómeno del soft law

35 Cfr. Thirlway, Hugh, "The Sources of International Law" en Evans, Malcolm (ed.), International Law, Oxford, 2002, pp. 117-144.

36 Cfr. Cassese, Antonio, International Law, Oxford University Press, 2002, pp. 115 y ss; González Campos, Julio; Sánchez Rodríguez, Luis y Sáenz de Santa María, Paz Andrés, Curso de derecho internacional público, 2a. ed., Civitas, Madrid, 2002, pp. 127 y ss.

37 Cfr. Evans, Malcolm (ed.), International Law, Oxford, University Press, 2003; Dixon, Martín \&McCorquodale, Robert, Cases \& Materials on International Law, 4th ed., Oxford University Press, 2003. 
pues incurre en una petición de principio pues supone el carácter absoluto del artículo 38, cuando, como ya se dijo, existe una amplia evidencia de que no es así, como lo demuestra la consideración de ciertos actos y comportamientos unilaterales estatales y determinadas resoluciones de organizaciones internacionales como generadores de obligaciones internacionales. ${ }^{38}$ De ahí que desde esta perspectiva, como considera Karl Zemanek 'the dichotomy of 'binding' and 'non-binding' is not really helpful to determining the nature of soft law". 39

El proceso de creación de derechos y obligaciones internacionales reposa en el consentimiento o en el consenso de los Estados, por tanto existe, utilizando la expresión de Baxter, una infinita variedad de manifestaciones del derecho internacional ${ }^{40}$ que no se constriñe al texto del artículo 38 .

Además, la creación de normas internacionales no supone una operación instantánea sino el desarrollo de un proceso complejo durante cierto periodo de tiempo, determinado por las circunstancias de formación y negociación, dependiendo el caso. El estudio tradicional del artículo 38 del Estatuto de la CIJ desde una perspectiva dicotómica conlleva a una concepción estática del derecho internacional contraria a la realidad que pretende explicar. La realidad internacional y la formación de normas internacionales se desenvuelven en el marco de un proceso dinámico en constante evolución. La atención debiera ponerse más que en el resultado en el proceso, en la dinámica de operación de la sociedad internacional, esto es, en el movimiento previo a la constatación de la existencia de la norma. Una teoría de la creación jurídica no supone la creación instantánea del derecho, una teoría sobre los procesos de creación normativa re-

38 Para ilustrar estos supuestos se suelen citar de la Corte Internacional de Justicia, respecto del valor de ciertas resoluciones internacionales, sus opiniones consultivas sobre la Legalidad de la amenaza o el empleo de armas nucleares de 8 de julio de 1996 y sobre las Consecuencias jurídicas para los Estados de la presencia continuada de Sudáfrica en Namibia (Sudoeste africano), no obstante la resolución 276 (1970) del Consejo de Seguridad, de 21 de junio de 1971; y su sentencia en el caso de las Actividades militares y paramilitares en Nicaragua y contra ésta (Nicaragua v. Estados Unidos de América) (fondo). Sentencia de 27 de junio de 1986. Respecto del valor de ciertos actos unilaterales las sentencias dictadas en los casos sobre los Ensayos Nucleares (Australia v. Francia) Sentencia de 20 de diciembre de 1974 y Templo de Preah Vihear, (fondo) Sentencia de 26 de mayo de 1961; y respecto del valor de ciertas declaraciones la sentencia de 5 de abril de 1933 dictada por la Corte Permanente de Justicia Internacional en el caso del Estatuto Jurídico de Groenlandia Oriental, Serie A/B, núm, 53.

39 Zemanek, "Is the term 'Soft law' convenient?", op. cit., p. 845.

40 Baxter, "International law in "her infinite variety", op. cit., p. 566. 
conoce que el derecho surge de la sociedad, a partir del desarrollo de ideas y la emergencia de nuevos valores que reclaman su reconocimiento formal y su protección jurídica. En consecuencia determinar un punto específico que permita diferenciar lo jurídico de lo "no-jurídico" no parece sencillo y tampoco deseable, pues implica una concepción reduccionista de procesos complejos. De ahí que se hable de la existencia de una "zona gris" que separa los valores sociales emergentes y las reglas de derecho bien establecidas. Esto supone que la frontera entre el derecho y el "no-derecho" o el "pre-derecho", la lex lata y la lex ferenda, no esta claramente delimitada y por tanto, el umbral del mundo jurídico no está del todo definido y quizá sea imposible e incluso inconveniente tratar de hacerlo. ${ }^{41}$

Parece que, debilitada la idea y el concepto mismo de las "fuentes" en el derecho internacional, se abre un abanico más rico de posibilidades y procesos de creación normativa que si bien, dada la naturaleza de la propia comunidad internacional, encuentran en los tratados y en la costumbre las formas principales de generación de derechos y obligaciones internacionales, también existen otros supuestos que no sólo deben verse como eslabones menores de una cadena que necesariamente derivaría en tratados o costumbre (aunque en efecto puede suceder así y así ha sucedido), sino que tienen un carácter independiente, en virtud de su propia naturaleza.

Si la puerta de entrada al mundo jurídico es difusa, esto no supone necesariamente que sea imposible identificar los diferentes momentos y las diferentes etapas que llevan a la consagración jurídica de los diferentes valores sociales. La "zona gris" es el ámbito donde se transforma la norma social en norma jurídica, como en la alquimia el opus nigrum, el mundo de lo jurídico también tiene sus formulas, el soft law es una de ellas y el acto de transformación y consagración que culmina el proceso de creación de lo jurídico, donde no existe duda sobre la existencia o no de un derecho y una obligación generalmente es el ámbito de la práctica internacional y nacional, cuando un juez o un árbitro internacional resuelve clarificar ese aspecto que hasta entonces era difuso o cuando los poderes públicos al interior de un Estado, en especial el ejecutivo o el judicial, deciden aplicar los instrumentos del soft law. Como estiman We-

41 Cfr. Abi-Saab, Geoges, "Les Sources du Droit International: Essai de Déconstruction", op. cit., pp. 37 y ss. 
llens y Borchardt: "The executive and judicial branch are the pre-eminent channels for the use and application of international soft law". ${ }^{42}$

Entendido así el soft law se integra a la explicación de un proceso más complejo de creación de derecho que aquel que supone la doctrina tradicional de las "fuentes" del derecho que enfatiza lo formal. ${ }^{43}$ Desde esta perspectiva, la dicotomía soft law/hard law se orienta hacia otras latitudes, donde la vaguedad del concepto cede terreno hacia un sentido más práctico: los mecanismos de cumplimiento de la normativa internacional y los distintos grados de responsabilidad internacional.

Como es bien sabido, la práctica internacional demuestra que el cumplimiento de las normas internacionales no está subordinado de forma exclusiva a la existencia de posibles sanciones derivadas de la violación a una norma internacional. ${ }^{44}$ En el ámbito internacional, la ausencia de una jurisdicción internacional obligatoria, el uso frecuente y en ocasiones excesivo de reservas y declaraciones interpretativas que limitan la competencia contenciosa de los tribunales internacionales, la pluralidad de mecanismos de solución de controversias internacionales, entre otras cosas, hacen que los mecanismos de adjudicación judicial internacional funcionen de forma excepcional y sólo operen de manera obligatoria en algunos regímenes especiales o subsistemas regionales. Asimismo, la exigibilidad de cumplimiento del derecho internacional basada en la reciprocidad entre sujetos se encuentra limitada por factores políticos y jurídicos, y las posibles contramedidas que pudiera adoptar un Estado o varios frente a otro por el incumplimiento de una obligación internacional también suponen limitaciones y circunstancias especiales dependiendo cada caso. ${ }^{45}$ En el ámbito estatal la aplicación de los tratados internacio-

42 Wellens, K. C y Borchardt, G. M., "Soft Law in European Community Law", European Law Review, October, 1989, p. 285.

43 El identificar los instrumentos del soft law exclusivamente con su carácter no vinculante (non-binding) desconoce los motivos que explican su existencia y en consecuencia las razones de su cumplimiento. Al respecto, entre otros: Zemanek, K., "The Legal Foundations of the International System. General Course on Public International Law", Recueil des Cours 1997, t. 266, 1998, pp. 141 y ss.

44 Cfr. Haas, Peter, "Choosing to Comply: Theorizing from International Relations and Comparative Politics" y Bilder, Richard, "Beyond Compliance: Helping Nations Cooperate" ambos en Shelton, Dinah (ed.), Commitment and compliance. The role of non-binding norms in the international legal system, op. cit., pp. 43-64 y 65-73.

$45 \mathrm{Al}$ respecto véase la sentencia de la Corte Internacional de Justicia en el Case Concerning the Gabcikovo-Nagymaros Project (Hungary/Slovakia), No. 92, 25 September 1997 (www.icj-cij. org) 
nales por los jueces naciones también se encuentra limitada por una serie de factores que van desde los métodos de incorporación del derecho internacional al derecho interno y la jerarquía de las normas internacionales, hasta el grado de independencia de la judicatura respecto del ejecutivo y la capacitación de los jueces en materias internacionales. ${ }^{46}$

Lo anterior supone que, pese a su carácter obligatorio, la exigibilidad de las normas internacionales (hard law) ante instancias judiciales (internacionales o nacionales) encuentra problemas que cuestionan precisamente su naturaleza hard, problemas que han merecido en no pocas ocasiones que se tache de inoperante, ineficaz y no jurídico al derecho internacional en su conjunto. Desgraciadamente para los negadores del derecho internacional, esta no es la única lectura posible de la realidad internacional. Lo cierto es que la gran mayoría de las normas internacionales gozan de amplio respaldo y espontáneo cumplimiento. ${ }^{47}$ Como afirma Karl Zemanek:

[I]f 'hard' international law is normally observed and applied, it is hardly because of the fear of eventual enforcement measures. Observance owes more to its general acceptance, to the recognition that the existing legal rules reflect the shared values and interests of the members of the international community and are, therefore, legitimate. Observance is thus rather motivated by the self-interest of States in securing a stable, safe and predicable world in which they can better pursue their broad political and economic goals. In other words: States have a shared interest in the maintenance of predictable behavior patterns and this reciprocal interest

46 Sobre la aplicación del derecho internacional por los jueces nacionales véase, entre otros: Knop, Karen, "Here and there: International Law in Domestic Courts", 32 N.Y.U.J.INT'L L. \& POL. 501 (2000); Conforti, Benedetto (repporteur), "The Activities of National Judges and the International Relations of their States, Preliminary Report", 65 Yearbook of the Institute of International Law (1993), Part. I; Falk, Richard, The Role of Domestic Courts in the International Legal Order, Syracuse University Press, New York, 1964.

47 Algunos autores sugieren que la afirmación del cumplimiento "espontáneo" del derecho internacional ha de tomarse con cautela. En palabras de Peter Haas: "The lawyers' dictum that 'must treaties are complied with most of the time' is surely premature, and probably exaggerated. Studies of compliance find variation in compliance along a number of dimensions. Not all countries comply with the same legal instruments, the same country may vary in its compliance with different legal instruments across functional areas and even within the same issue area, and such patterns may change over time". Haas, Peter, "Choosing to Comply: Theorizing from International Relations and Comparative Politics", en Shelton, Dinah (ed.), Commitment and compliance. The role of non-binding norms in the international legal system, cit., p. 44. 
contributes more to the performance of obligations than the possibility of sanctions. $^{48}$

De esta forma el carácter hard o soft de determinada norma no está directamente relacionado con el grado de su cumplimiento. Normas soft pueden gozar de alto prestigio, amplio consenso y espontánea observancia; mientras normas hard pueden requerir de muchos esfuerzos diplomáticos o largos procesos jurisdiccionales antes de alcanzar su pleno cumplimiento, o incluso resultar imposible la exigibilidad del mismo. De igual manera, en los procedimientos de solución de controversias, los órganos encargados de resolver las diferencias, en especial los de naturaleza jurisdiccionales, toman en consideración no sólo instrumentos del denominado hard law sino también instrumentos del soft law que se encuentran relacionados con los derechos y obligaciones en conflicto. No obstante lo anterior, ha de tomarse en cuenta que el derecho internacional no es un simple agregado o conjunto de normas sino un sistema normativo que no sólo tiene por objeto la resolución de controversias, sino y quizá prioritariamente, promover y asegurar ciertos valores que se consideran fundamentales para la convivencia de la comunidad internacional. ${ }^{49}$

Esto nos remite nuevamente a la concepción de un orden internacional mucho más abierto, dinámico, complejo y diverso, entendido más como un proceso en constante transformación que como un simple conjunto de normas ya dado donde sólo es preciso identificar la validez de sus normas para ser aplicadas por los operadores jurídicos en la solución de una controversia internacional.

\section{LAS FORMAS DEL SOFT LAW}

Como lo reconoce la mayor parte de la doctrina, no es sencillo precisar el significado de la expresión soft law, así como tampoco establecer su alcance y contenido. Su significado depende del concepto que se tenga del derecho internacional. El término es usualmente empleado por la doctrina para describir principios, reglas, estándares o directrices que carecen de efecto vinculante aunque no dejan por ello de producir determi-

48 Zemanek, K., "Is the term 'Soft law' convenient?", op. cit., p. 856.

49 Higgins, Rosalyn, Problems and Process. International Law and How we use it, Clarendon Press-Oxford, 1996, p. 1. 
nados efectos jurídicos. ${ }^{50}$ Daniel Thürer considera al soft law "as a phenomenon in international relations covers all those social rules generated by States or other subjects of international law which are not binding but which are nevertheless of special legal relevance". ${ }^{51}$

Este fenómeno envuelve una amplia gama de documentos internacionales: resoluciones de organizaciones internacionales, recomendaciones e informes adoptados por organismos internacionales o dentro de conferencias internacionales; programas de acción; textos de tratados que no han entrado en vigor, declaraciones interpretativas de determinados tratados o convenios; disposiciones programáticas o non-self-executing; acuerdos no normativos, acuerdos políticos o gentlemen 's agreement, códigos de conducta, directrices, estándares, etc. ${ }^{52}$

La diversidad de instrumentos internacionales que suelen enmarcarse dentro del denominado soft law hacen de él un concepto demasiado amplio para dotarlo de un sentido único en el discurso del derecho internacional. Tal parece que el dato unificador es aquel que se refiere a sus efectos no obligatorios y que lo define por contraposición al denominado hard law. En este contexto de incertidumbre, Daniel Thürer identifica cuatro aspectos que resultan apropiados para describir el concepto de soft law, a saber:

50 Cfr. entre otros: Sztucki, Jerzy, "Reflections on International "Soft law", Ramberg, Jan, et al. (eds), Festskrift till Lars Hjerner. Studies in International Law, Norstedts, Stockholm, 1990, pp. 549-575. Sztucki distingue dos posibles significados para la expresión "soft law". La primera se refiere al "contenido" de determinados instrumentos internacionales en principio obligatorios (hard law) pero que dependen para su aplicación de ciertos mecanismos de implementación o que constituyen cláusulas generales de cumplimiento progresivo. En segundo lugar el término es empleado para expresar la "naturaleza" de determinados instrumentos internacionales que no tienen carácter obligatorio, como son la mayoría de las resoluciones de las organizaciones internacionales o de ciertos acuerdos políticos internacionales como el Acta Final de Helsinki (pp. 551-554).

51 Thürer Daniel, "Soft Law", op. cit., p. 454.

52 Incluso en ciertas materias como los derechos humanos se distingue entre "Primary Soft law" y "Secundary Soft law". En palabras de Dinah Shelton: "Primary soft law can be considered as those normative texts not adopted in treaty form that are addressed to the international community as a whole or to the entire membership of the adopting institution or organization. Such an instrument may declare new norms, often as an intended precursor to adoption of a later treaty, or it may reaffirm or further elaborate norms previously set forth in binding or non-binding texts... Secondary soft law includes the recommendations and general comments of international human rights supervisory organs, the jurisprudence of courts and commissions, decisions of special rapporteurs and other ad hoc bodies, and the resolutions of political organs of international organization applying primary norms". Shelton, "Commentary and conclusions", en Shelton, Dinah (ed.), Commitment and Compliance. The Role of Non-binding Norms in the International Legal System, cit., pp. 449-450. En la misma obra Chinkin, "Normative Development in the international legal system", pp. 26 y 27. 
Firstly, soft law does generally express common expectations concerning the conduct of international relations as it is often shaped by or arise within the framework of international organizations. Secondly, soft law is created by subjects of international law... Thirdly, soft law rules have not - or not entirely - passed through all stages of the procedures prescribed for international law-making; they do not stem from a formal source of law and thus lack legal binging force. Fourthly, soft law — despite its legally noncommittal quality - is characterized by a certain proximity to the law and above all by its capacity to produce certain legal effects. ${ }^{53}$

En este sentido, el soft law resulta especialmente relevante cuando el principio de buena fe encabeza las relaciones interestatales, así como en el desarrollo, interpretación y clarificación del derecho internacional o del derecho estatal por los operadores jurídicos. En palabras del propio Thürer: "Soft law is of particular importance where good faith needs to be protected. It may also prove helpful in developing; interpreting and clarifying international as well as municipal law". Sobre esta base, Thürer, como gran parte de la doctrina, considera tres instrumentos del soft law: las resoluciones, recomendaciones y decisiones de organizaciones internacionales; los acuerdos no normativos (Non-binding international legal agreements); y las partes no obligatorias de los acuerdos vinculantes (tratados). ${ }^{54}$

Otros autores, sin embargo matizan el hecho de que sólo los sujetos de derecho internacional participen en la elaboración de instrumentos del soft law. Para Wolfgang Reinicke y Jan Martín Witte, por ejemplo, una características de los acuerdos internacionales no normativos es servir como herramienta para establecer vínculos entre instituciones públicas y entidades privadas transnacionales y no gubernamentales. ${ }^{55}$ En este senti-

53 Thürer, D., "Soft Law", op. cit., p. 454.

54 Ibidem, p. 460. Thürer considera que la distinción entre derecho y "no-derecho" no debe abandonarse $\mathrm{y}$, por tanto, los instrumentos del soft law no deben considerarse como una fuente independiente del derecho internacional. Sin embargo, el concepto de soft law representa un fenómeno importante en las relaciones internacional que a pesar de carecer de obligatoriedad no deja de producir ciertos efectos jurídicos. En los últimos años los instrumentos del soft law han comenzado a jugar un rol cada vez más importante en las relaciones internacional. En el marco de las conferencias internacionales, determinados principios y directrices políticas adoptadas por el consenso de los participantes tienen gran relevancia y expresan un alto grado de sensibilidad y voluntad política de los miembros de la comunidad internacional, principalmente, en aquellos temas que manifiestan problemas comunes de la humanidad en su conjunto.

55 Reinicke, Wolfgang, H. y Martin Witte, Jan, "Interderpendence, Globalization, and Sovereignty: The Role of Non-binding International Legal Accords", en Shelton, Dinah (ed.), Commit- 
do, en la elaboración de instrumentos del soft law pueden participar sujetos de derecho internacional exclusivamente; tales sujetos conjuntamente con otros actores internacionales e incluso actores privados, grupos de expertos o individuos. ${ }^{56}$ Desde esta perspectiva, el hecho de que los instrumentos de soft law estén abiertos a otros actores internacionales es uno de sus datos característicos y por supuesto uno de sus aspectos más controvertidos.

Dada la complejidad del fenómeno, el soft law se presenta, utilizando la expresión de Baxter, ${ }^{57}$ en una infinita variedad de formas. En general se reconocen cuatro instrumentos: las resoluciones no obligatorias de las organizaciones internacionales, los acuerdos interestatales no normativos o no vinculantes jurídicamente, el soft law de los actores no estatales y el denominado "soft law material" ${ }^{58}$ Este último término está referido al contenido de los instrumentos internacionales, de tal forma que un tratado internacional puede contener disposiciones vagas o indeterminadas, establecer obligaciones de carácter programático o declaraciones generales y, por tanto, tener un contenido materialmente soft que no establezca obligaciones para las partes.

Esta categoría es sin duda la más polémica porque supone que un instrumento en principio obligatorio (como es un tratado internacional) contiene disposiciones no obligatorias. Lo cierto es que las obligaciones de un tratado se determinan a partir de su contenido y nada impide que un tratado establezca obligaciones mínimas o programáticas, lo que no significa que no deban cumplirse o que carezcan de fuerza vinculante., sino solamente la existencia de diferentes obligaciones (por ejemplo, obligaciones de comportamiento u obligaciones de resultado) o de dispo-

ment and Compliance. The Role of Non-binding Norms in the International Legal System, cit., pp. 94 y 95.

56 Cfr. Chinkin, C. M., "The challenge of soft law: Development and change in international law", International and Comparative Law Quarterly, October, 1989, p. 851, y "Normative Development in the international legal system", en Shelton, Dinah (ed.), Commitment and compliance. The role of non-binding norms in the international legal system, cit., pp. 29 y 34 y ss.; Mazuelos, "Soft Law: ¿Mucho ruido y pocas nueces?”, op. cit., p. 13.

57 Baxter, "International law in "her infinite variety", op cit, p. 566.

58 Cfr. Mazuelos, A., "Soft Law:¿Mucho ruido y pocas nueces?”, op. cit., pp. 3 y ss; Weil, Prosper, "Le Droit International en quête de son identité. Cours général de droit international public", Recueil des Cours 1992, VI, t. 237, The Hague-Boston-London, 1996, pp. 215 y ss; Ida, Ryuichi, "Formation des Normes Internationales dans un Monde en Mutation Critique de la Notion de Soft Law”, en Mélanges Michel Virally, Paris, 1991, 333-340. 
siciones autoejecutables o no autoejecutables (non-selfexecuting), pero en modo alguno la ausencia de obligaciones jurídicas. ${ }^{59}$

Por ello, el denominado "soft law material" es el que más se presta a generar polémicas de tipo ideológico, a encubrir intereses políticos o a justificar unilateralismos que suponen la inexistencia de obligaciones internacionales dentro de instrumentos que tienen por objeto precisamente crear obligaciones entre las partes. Una interpretación de este tipo sería incompatible con el objeto y fin del tratado, salvo que estemos en presencia de acuerdos no normativos, lo que supondría otro instrumento del soft law. ${ }^{60}$ Por todo ello, no es conveniente identificar disposiciones de tratados como normas de soft law con base exclusivamente en su carácter general o programático.

\section{CARACTERÍSTICAS GENERALES DE LOS INSTRUMENTOS DEL SOFT LAW}

Los instrumentos de soft law se caracterizan por ser documentos que reflejan la tendencia actual de la comunidad internacional por una mayor

59 Por poner sólo un ejemplo, las obligaciones programáticas establecidas en el Pacto Internacional de Derechos Económicos Sociales y Culturales, en el sentido de que cada Estado "se compromete a adoptar medidas... hasta el máximo de los recursos de que disponga, para lograr progresivamente, por todos los medios apropiados... la plena efectividad de los derechos reconocidos" en el Pacto (art. 2.) han sido interpretadas por el Comité de Derechos Económicos, Sociales y Culturales, como obligaciones generales que incluyen tanto obligaciones de comportamiento como obligaciones de resultado y que la expresión "progresiva efectividad" no se ha de interpretar equivocadamente "como que priva a la obligación de todo contenido significativo"; por el contrario, debe interpretarse "a la luz del objetivo general, en realidad razón de ser, del Pacto, que es establecer claras obligaciones para los Estados Partes con respecto a la plena efectividad de los derechos de que se trata. Este impone así una obligación de proceder lo más expedita y eficazmente posible con miras a lograr ese objetivo." Cfr. Comité de Derechos Económicos, Sociales y Culturales, Observación General No. 3 sobre La indole de las obligaciones de los Estados Partes (párrafo 1 del artículo 2 del Pacto), Quinto Periodo de sesiones (1990), en Doc. E/1991/23 o HRI/GEN/1/Rev.5, pp. 19 y ss.

60 Al respecto, véase: Remiro Brotóns, Antonio, "De los tratados a los acuerdos no normativos", en Ministerio de Asuntos Exteriores, La celebración de tratados internacionales por España: problemas actuales, Madrid, 1990, pp. 23-48. El profesor Remiro propone un decálogo con los "mandamientos" que debería seguir quien quiera celebrar un acuerdo no normativo, entre ellos destacan: no denominar al acuerdo tratado, convención o convenio, siendo preferible memorándum (de entendimiento), programa /de actuación) o minuta; utilizar para los predicados de conducta expresiones "de baja intensidad"; no formular reservas ni declaraciones interpretativas; no inscribir el texto en el Registro de Tratados y Acuerdos Internacionales de la Secretaría General de Naciones Unidas. 
interrelación, interdependencia y globalización. ${ }^{61}$ En este contexto las organizaciones internacionales, junto con otros actores internacionales, buscan mediante estos instrumentos promover acciones en materias de interés general en temas relativamente nuevos para la comunidad internacional en los cuales (por diferentes intereses políticos, económicos o de otra naturaleza) es difícil llegar a un acuerdo general con carácter obligatorio entre los Estados. ${ }^{62}$ Por ello, tales instrumentos no imponen obligaciones internacional; sin embargo, esto no significa que carezcan de todo efecto jurídico, pues en diversas ocasiones reflejan el estado del desarrollo de normas consuetudinarias o son la base de tratados futuros e incluso, por su propia naturaleza, en tanto generan expectativas de comportamiento, están regidos de alguna manera por el principio de la buena fe, lo que explica su utilidad y cumplimiento. ${ }^{63}$

En este sentido, se deben tomar en cuenta algunos elementos comunes a los distintos instrumentos que se asocian con el concepto de soft law. Tales instrumentos expresan expectativas comunes de conducta adoptadas generalmente en el marco de organizaciones internacionales y son creados o adoptados en su mayoría por sujetos de derecho internacional (con la participación o no de otros actores internacionales), sin que para ello se siga todo el proceso tradicional para la creación de normas internacionales, por lo que no pueden considerarse como "fuentes formales de derecho" (tratados o costumbre) y, en consecuencia, carecen de fuerza obligatoria. Sin embargo, y este es el principal aspecto distintivo, a pesar de carecer de fuerza vinculante tales instrumentos se caracte-

61 Reinicke, Wolfgang, H. y Martin Witte, Jan, "Interdependence, Globalization, and Sovereignty: The Role of Non-binding International Legal Accords" y O'Connell, Mary Ellen, "The role of Soft Law in a Global Order", ambos en Shelton, Dinah (ed.), Commitment and Compliance. The Role of Non-binding Norms in the International Legal System, op. cit., pp. 76-114.

62 Piénsese, por ejemplo, en la protección al medio ambiente, el derecho al desarrollo o ciertos ámbitos de los derechos humanos como los derechos económicos, sociales y culturales; aunque también en ciertos aspectos comerciales, financieros y de control de armamento. Cfr. Hey, Ellen, "Hard Law, Soft Law, Emerging International Environmental Law and the Ocean Disposal Options for Radioactive Waste", en Netherlands International Law Review, WL, 405-448, 1993; Fitzmaurice, Malgosia, "International Protection of the Environment", Recueil des Cours 2001, t. 293, 2002, pp. 9-488. En general sobre estos temas: Shelton, Dinah, (ed.) Commitment and compliance. The role of non-binding norms in the international legal system, op. cit.. La segunda parte del libro contiene diferentes trabajos que desarrollan temas específicos respecto a cuestiones ambientales, comercio y finanzas, derechos humanos y control de armas.

63 Cfr. Cassese, Antonio, International law, op. cit., pp. 160-161; Shelton, Dinah, "Law, Non-law and the problem of 'Soft Law", en Shelton, D., (ed.), Commitment and compliance. The role of non-binding norms in the international legal system, op. cit., p. 3. 
rizan por su capacidad de producir ciertos efectos jurídicos que varían dependiendo el grado de compromiso, la precisión de sus cláusulas y la delegación de facultades a organismos internacionales de supervisión. ${ }^{64}$

Respecto a los criterios para determinar la existencia de un determinado instrumento del soft law, Christine Chinkin identifica los siguientes: $i)$ que sean formulados en términos no obligatorios de acuerdo a los procesos tradicionales de creación de derechos ( $v . g r$. tratado o costumbre); ii) contengan términos vagos e imprecisos; iii) procedan de órganos carentes de autoridad para crear normas internacionales; iv) estén dirigidos a actores no estatales, cuya práctica no pueda configurar una costumbre internacional; v) permanezcan ajenos a cualquier teoría de la responsabilidad internacional; vi) se basen exclusivamente en la adhesión voluntaria y no exista un mecanismo jurídico para su exigencia. Otro posible modelo de clasificación de los instrumentos del soft law se basa en la relación que guardan con los correspondientes del hard law. En este sentido se distingue entre soft law explicativo o interpretativo del hard law; hard law emergente en la forma de soft law; soft law como evidencia de obligaciones jurídicas, soft law paralelo al hard law; y soft law como fuente de obligaciones internacionales. En palabras de la profesora Chinkin:

i) Elaborative soft law, that is principles that provide guidance to the interpretation, elaboration, or application of hard law...

ii) Emergent hard law, that is principles that are first formulated in non-binding form with the possibility, or even aspiration, of negotiating a subsequent treaty, or harden into binding custom through the development of state practice and opinio juris...

iii) Soft law as evidence of the existence of hard obligations.

iv) Parallel soft and hard law; that is similar provisions articulated in both hard and soft forms allowing the soft version to act as a fallback provision.

v) Soft law as a source of legal obligations, trough acquiescence and estoppel, perhaps against the original intentions of the parties. ${ }^{65}$

64 Cfr. Abbott y Snidal, "Hard and soft law in international governance", op. cit., p. 424.

65 Chinkin, C., "Normative Development in the international legal system", en Shelton, Dinah (ed.), Commitment and compliance. The role of non-binding norms in the international legal system, op. cit., pp. 30 y 31. 
Sin embargo, como considera la misma profesora Chinkin, esta categoría si bien es útil es problemática en tanto define al soft law en términos de su relación con el hard law y no en sus propios términos, lo que excluye lo que podría llamarse "soft law autónomo" ('autonomous soft law). Asimismo, esta clasificación supone que la transformación en hard law es deseable, lo que no siempre es así, por ejemplo cuando cierta flexibilidad es preferible para llegar a determinados consensos.

Por ello, conviene distinguir aquellos supuestos en que los diferentes actores internacionales deliberadamente escogen instrumentos del soft law, sea por considerar su flexibilidad y falta de exigibilidad como una ventaja o por representar la única forma posible de establecer ciertos acuerdos, estándares, directrices o políticas comunes.

\section{LAS RAZONES DEL SOFT LAW Y SUS CONSECUENCIAS}

El desarrollo del soft law internacional se explica por distintas razones, algunas de carácter estructural de la sociedad internacional, y otras por razones de tipo político e ideológico que no siempre son fáciles de reconocer e identificar. Como se reconoce generalmente por la doctrina, el fenómeno del soft law surgió en los años setenta como respuesta a los nuevos planteamientos teóricos motivados por el denominado nuevo orden económico internacional; en el marco de la revisión de la teoría tradicional de las fuentes del derecho internacional y del papel de las resoluciones de las organizaciones internacionales. En la actualidad el fenómeno se extiende ampliamente por diversos ámbitos del derecho internacional. ${ }^{66}$

Entre las causas del incremento en la adopción de instrumentos propios del soft law está el debilitamiento del proceso de codificación y desarrollo progresivo llevado a cabo en el seno de la Comisión de Derecho Internacional (CDI) que en ocasiones ha puesto en duda la efectividad del proceso de codificación y el riesgo de someter determinados instrumentos a quirúrgicos procesos de negociación y ratificación ("codifica-

66 Véase, entre otros: Wellens, K. C y Borchardt, G. M., "Soft Law in European Community Law", European Law Review, October, 1989, p. 267, y Shelton, Dinah (ed.), Commitment and compliance. The role of non-binding norms in the international legal system, op. cit. 
ción inversa"). ${ }^{67} \mathrm{Sin}$ embargo, tales instrumentos ofrecen sus propias ventajas, pues reducen los costos propios de las negociaciones convencionales, así como el impacto que la delegación de facultades a órganos supranacionales podría tener en el ámbito de la soberanía. Evitan la revisión parlamentaria en el derecho interno y excluyen los riesgos derivados del incumplimiento y las consecuencias de la responsabilidad internacional en el marco de los mecanismos institucionales de solución de controversias. En ocasiones es el único recurso disponible para las instituciones internacionales. Además, la creciente adopción de instrumentos de soft law en las conferencias internacionales permite la participación de actores no estatales, tales como las organizaciones no gubernamentales, lo que en ocasiones otorga más legitimidad a las normas derivadas de tales procesos. De esta forma, en ciertos casos a la falta de obligatoriedad directa se contrapone la legitimidad y el amplio consenso del soft law por lo que los Estados, si bien no están formalmente vinculados por tales disposiciones, cumplen tales expectativas de comportamiento en busca de reconocimiento, credibilidad y confianza de los actores internacionales, aunque, por supuesto, se entreabre la puerta para el informalismo propio del relativismo estatal. ${ }^{68}$

67 El éxito de algunos de los primeros proyectos de la CDI, como aquellos en materia de derechos de los tratados o sobre el derecho diplomático y consular, no se corresponde con algunos otros que no han recibido de la comunidad internacional la respuesta deseada y no han alcanzado el número de ratificaciones necesarias para su entrada en vigor. Ejemplo de tal crisis es la discusión entorno a la forma final que adaptará el Proyecto de artículos sobre responsabilidad del estado por hechos internacionalmente ilícito adoptado por la CDI en 2001, después de casi medio siglo de preparación y cinco relatores especiales, pues, dada su importancia, un sinuoso proceso de negociación y ratificación podría restar efectividad a los artículos del proyecto y vulnerar su carácter codificador de normas consuetudinario en la materia; siendo muy posible que ingrese a las filas del "derecho blando" o soft law en forma de declaración o alguna otra que le permita conservar su efectividad en la practica internacional. De ser el caso, ¿esto demerita el contenido normativo del proyecto? Creo que no, al contrario, lo aclara y en cierto sentido lo hace posible. Aunque lo deseable sería una convención general adoptada sin reservas por la gran mayoría de los Estados. Sobre este debate véase el propio informe de la Comisión de Derecho Internacional en su 53o. periodo de sesiones del 2001 en su parte relativa al Proyecto de Artículos sobre Responsabilidad del Estado por Hechos Internacionalmente Ilícitos. A/CN.4/SR.2662, cap. IV, párrs. 61 y ss. Disponible en www.un.org/law/ilc/reports/2001/2001report.htm. La Asamblea General de las Naciones Unidas tomó nota de los artículos sobre responsabilidad y los señaló a la atención de los Estados mediante su resolución 56/83 adoptada el 12 de diciembre de 2001.

68 Cfr. Shelton, D., "Law, Non-law and the problem of 'Soft Law", en Commitment and compliance. The role of non-binding norms in the international legal system, op. cit., pp. 12 y ss; Chinkin, C. M., "The challenge of soft law: Development and change in international law", op. cit., pp. 859 y ss. 
Otro aspecto importante a considerar cuando se habla de soft law es el hecho de que aún a falta de mecanismos institucionales de control y cumplimiento, tales instrumentos pueden producir efectos en el ámbito interno. Por ejemplo, pueden servir de inspiración a parlamentos para la adopción de legislación interna o a los jueces como pautas interpretativas de tratados internacionales, para determinar la existencia de una costumbre o un principio general de derecho internacional. En este sentido, es importante destacar la transformación del soft law en hard law, no sólo en el ámbito internacional a través de la celebración de tratados posteriores o de la configuración de una práctica consuetudinaria, sino también desde el punto de vista interno con la adopción de medidas legislativas o judiciales que incorporan los estándares del soft law a la normatividad interna reconociéndole carácter obligatorio. ${ }^{69}$ En consecuencia, como apunta Dinah Shelton:

Despite their limited juridical effect, non-binding instruments have an essential and growing role in international relations and in the development of international law. Such instrument may (1) precede and help from international customary and treaty law; (2) fill in gaps in international legal instruments and further define existing custom; (3) form part of the subsequent State practice that can be utilized to interpret treaties; and (4) substitute for legal obligation when on-going relations make formal treaties too costly and time-consuming or otherwise unnecessary. In the first three categories, non-binding instruments are often linked in one way or another to binding ones. The last category is perhaps the most interesting, because the extent to which members of the international community are willing to accept informal commitments and non-binding expressions of expected behavior in their relations with others may reflect a maturing of the legal system and international society. ${ }^{70}$

69 Véase, entre otros: Boyle, A .E., "Some reflections on the relationship of treaties and soft law", International and Comparative Law Quarterly, vol. 48, October, 1999, pp. 901-913; Chinkin, C. M., "The challenge of soft law: Development and change in international law", op. cit., pp. 850-866; Elias, Olufemi y Lin, Chin, "General Principles of Law, 'Soft' Law and the Identification of International Law, Netherlands Yearbook of International Law, vol. XXVIII-1997, pp. 3-49.

70 Shelton, Dinah, "International law and 'Relative Normativity", en Evans, Malcolm (ed.), International Law, Oxford, University Press, 2003, p. 160. 


\section{COMENTARIO FINAL. EL SOFT LAW EN TIEMPOS DE UNILATERALISMOS}

La sociedad internacional es un sistema complejo de interrelaciones políticas, económicas y culturales que no se reduce a la producción de normas jurídicas; asimismo, el derecho es un sistema en cuya estructura discursiva conviven diferentes enunciados normativos que no necesariamente producen los mismos efectos. Esto nos lleva a concluir que no todos los problemas en la sociedad internacional deben resolverse y se resuelven mediante compromisos jurídicos ante la CIJ u otros mecanismos institucionales de solución de controversias, así como tampoco el sistema jurídico es un discurso cerrado a otros enunciados, distintos a las normas propiamente dichas, que produzcan determinados efectos jurídicos.

En este sentido, el soft law cumple una función propia dentro del discurso jurídico. Con el uso del término no sólo se pretende evidenciar la existencia de determinados instrumentos internacionales que no obstante no ser vinculantes tienen relevancia jurídica, sino también albergar bajo su manto diversas manifestaciones de acuerdos interestatales y consensos internacionales que independientemente de su valor jurídico se incorporan al discurso internacional y producen ciertos efectos que repercuten de diferentes formas en la formación, desarrollo, interpretación, aplicación y cumplimiento del derecho internacional, tanto en el ámbito interno de los Estados como en el propio seno del derecho internacional. Es el caso por ejemplo, de los programas de acción adoptados en el marco de las conferencias internacionales.

Si la función principal del término soft law fuere esclarecer el valor jurídico de determinados instrumentos internacionales (por ejemplo, las resoluciones de organismos internacionales) o identificar determinados acuerdos como jurídicamente relevantes (por ejemplo, programas de acción, acuerdos no normativos, códigos de conducta, etc.) quizá sería mejor referirse a cada uno de ellos por su nombre y función y no englobarlos en una categoría que en sí misma carece de precisión. La función del soft law no es, evidentemente, aclarar conceptos, pues tendría que empezar por aclararse a sí mismo (cuestión todavía no del todo resuelta por la doctrina internacional). ${ }^{71}$

71 Aunque es preciso reconocer que en el discurso del derecho internacional encontramos muchos otros términos imprecisos como "diplomacia parlamentaria", "legislación o ley 
Reconocer que el empleo del término soft law responde a una necesidad práctica más que lingüística, que su uso común busca explicar el funcionamiento del sistema internacional en su conjunto más que el de ciertas normas sociales, y que su utilidad deriva más de su propia ambigüedad que de su nitidez, es reconocer al mismo tiempo que el derecho internacional como todo ordenamiento jurídico está expuesto a los juegos del lenguaje, que el valor jurídico y el valor persuasivo operan conjuntamente y que en el fondo la realidad del derecho es una realidad que se construye a partir de determinados referentes teóricos y expresiones prácticas que se encuentran en constante reacomodo y transformación.

Aunque la discusión en torno al soft law se ubica en el denominado ámbito de las "fuentes" del derecho internacional, el empleo del término no tienen por objeto necesariamente establecer nuevos mecanismos de producción normativa ni resolver si los enumerados en el artículo 38 del Estatuto de la CIJ son los únicos posibles, deseables o existentes. Tampoco el término ha de resolver la problemática derivada de los límites entre el derecho internacional y la política internacional, aunque su habitad sea precisamente esa zona gris que divide el mundo de lo fáctico, lo ético, lo político, lo jurídico, lo teórico, lo práctico, lo concreto y lo programático. El soft law representa el primer eslabón de una cadena que va desde lo soft hasta lo hard y que culmina con las normas imperativas o ius cogens internacional.

En consecuencia, los instrumentos propios del denominado soft law se caracterizan por ser un conjunto abierto y flexible que no necesariamente se opone al hard law, ni pretende sustituirlo, sino que en muchas ocasiones lo antecede o lo complementa. Su naturaleza "informal" permite adaptar el sistema jurídico a los complejos desarrollos de los procesos de globalización mundial y abre espacios de participación a otros actores que están excluidos de los procesos formales de creación normativa. Esto otorga legitimidad al sistema y presiona políticamente a los

internacional", o el propio "ius cogens". En opinion de Jerzy Sztucki: "While the use of adequate terminology is, of course, a virtue of the legal profession, there appears to be nothing basically wrong in using handy capsule formulas — even if not quite adequate - to denote rather complex phenomena. For, "soft law" appears to be such a formula, denoting the body of international instruments but which per se do not make law (i. e., are of non-legal character) but which still possess — variable - regulatory force and even juridically are not irrelevant insofar as they can —again, in varying degree - reflect a stage in the process of formation of legal norms... "Soft law" is not the only not quite adequate term in circulation [p. e] 'international legislation"'. Sztucki, Jerzy, "Reflections on International "Soft law", op. cit., p. 573. 
Estados que prefieren cumplir de buena fe sus "compromisos" en materias de interés general para la comunidad internacional.

A pesar de lo anterior, debemos reconocer que, como todo concepto ambiguo, el soft law tiene muchas lecturas. En el escenario internacional actual, marcado por el unilateralismo de ciertas potencias (en particular de los Estados Unidos) el uso caprichoso del soft law puede significar la banalización de muchos ámbitos del derecho internacional (por ejemplo, protección del medio ambiente, respeto a los derechos humanos, limitación de armamento, etc.) y abrir la puerta al informalismo bajo el pretexto de la no vinculación de sus disposiciones (en especial cuando se utiliza el término "soft law material" para referir el contenido de determinadas cláusulas de acuerdos vinculantes) independientemente de que en el momento de su adopción los Estados manifestaran su consentimiento y se vanagloriaron de su adopción. Sin embargo, a pesar de ello, creemos que el concepto de soft law permite explicar un fenómeno generalizado que refleja el comportamiento de diversos actores internacionales y a su vez muestra la amplia posibilidad de adaptación del derecho internacional. Si algunos instrumentos de soft law son fuentes de derechos y obligaciones internacionales es algo que tiene que definirse en cada caso, lo que es innegable es que el fenómeno existe y debe incorporarse a los cursos y programas de estudio del derecho internacional pues, en definitiva, tiene ya un lugar reconocido en la doctrina internacional.

En conclusión, en mi concepto, se obtienen más ventajas buscando una descripción del soft law como fenómeno de la sociedad internacional y analizándolo en todas sus posibilidades y consecuencias que desechando el concepto por su carácter ambiguo, redundante y poco claro. Pues, si bien desde tiempo atrás se estudia el efecto de las resoluciones de las organizaciones internacionales y de otros actos no vinculantes, tales como, los acuerdos no normativos, acuerdos políticos o gentlemen's agreements, en mi concepto la idea del soft law, a pesar de su redundancia, permite analizar el fenómeno como un acontecimiento cultural que refleja el estado actual del funcionamiento de la sociedad internacional y nos muestra sus diferentes posibilidades incluso aquellas que, en sus extremos, podrían afectar el funcionamiento del sistema y fraccionar aún más a la comunidad internacional. Esta es la doble cara del soft law que, en el fondo, caracteriza la estructura del derecho internacional, donde legalidad y legitimidad se mantienen en un constante "equilibrio precario" siempre al borde aunque también siempre de pie. 


\section{BIBLIOGRAFÍA}

ABI-SAAB, Geoges, "Les Sources du Droit International: Essai de Déconstruction", en El derecho internacional en un mundo en transformación, Liber Amicorum en homenaje al professor Eduardo Jiménez de Aréchaga, Fundación de Cultura Universitaria, Uruguay, 1994, t. 1.

ABBOTT, Kenneth W. y SNIDAL Duncan, "Hard and soft law in international governance", en International Organization, vol. 54, 2000.

American Society of International Law Proceedings, 82 Am. Soc'y Int'1 L. Proc (1988).

BARBERIS, Julio, "Los actos jurídicos unilaterales como fuente del derecho internacional público", en Hacia un nuevo orden internacional y europeo. Estudios en homenaje al profesor don Manuel Diez de Velasco, Madrid, Tecnos, 1993.

_- Formación de derecho internacional, Buenos Aires, Ábaco Rodolfo de Palma, 1994.

BAXTER, R. R., "International law in "her infinite variety", International and Comparative Law Quarterly, vol. 29, October, 1980.

BOYLE, A .E., "Some reflections on the relationship of treaties and soft law", en International and Comparative Law Quarterly, vol. 48, October, 1999.

Brownlie, Ian, Principles of Public International Law, 4a. ed, Oxford Clarendon Press, 1990

CASANOVAS Y LA ROSA, Oriol, "Unidad y pluralismo en derecho internacional público", Curso Euromediterraneo Bancaja de Derecho Internacional, vol. II, 1998.

CASSESE, Antonio, International Law, New York, Oxford University Press, 2002.

ChInKIN, Christine M., "The challenge of soft law: Development and change in international law", International and Comparative Law Quarterly, October, 1989.

- "Normative Development in the international legal system", en Shelton, Dinah (ed.), Commitment and compliance. The role of non-binding norms in the international legal system, New York, Oxford University Press, 2000.

DuPuY, Pierre-Marie, "Théorie des Sources et Coutume en Droit International Contemporain", en El Derecho internacional en un mundo 
en transformación, Liber Amicorum en homenaje al profesor Eduardo Jiménez de Aréchaga, Uruguay, Fundación de Cultura Universitaria, 1994, t. 1.

ELIAS, Olufemi y LiN, Chin, “General Principles of Law, 'Soft' Law and the Identification of International Law, Netherlands Yearbook of International Law, vol. XXVIII-1997.

FASTENRATH, Ulrich, "Relative Normativity in International Law", 4 European Journal of International Law, 1993.

FitzMAURICE, Malgosia, "International Protection of the Environment", Recueil des Cours 2001, t. 293, 2002.

GonzÁlez CAMpos, Julio; SÁnChez RodríGuez, Luis y SÁEnZ DE SAnta MARÍA, Paz Andrés, Curso de derecho internacional público, 2a. ed., Madrid, Civitas, 2002.

GutiÉRreZ EsPaDA, Cesáreo, Derecho internacional público, Madrid, Trotta, 1995.

Henkin, Louis; Crawford Pugh, Richard; Shachter, Oscar y SMIT, Hans, International law: cases and materials, 3a. ed., St. Paul, Minn, West Publishing co., 1993.

HEY, Ellen, "Hard Law, Soft Law, Emerging International Environmental Law and the Ocean Disposal Options for Radioactive Waste", en Netherlands International Law Review, WL, 1993.

HiLlGENBERG, Hartmut, “A Fresh Look at Soft Law”, European Journal of International Law, núm. 3, 1999.

Hoof, G. J. H. van, Rethinking the Sources of International Law, The Netherlands, Kluwer Law, 1983.

IDA, Ryuichi, "Formation des Normes Internationales dans un Monde en Mutation Critique de la Notion de Soft Law", Mélanges Michel Virally, Paris, 1991.

KLABBERS, Jan, "The redundancy of soft law", Nordic Journal of International Law 65, 1996.

LAGOS, Enrique, "Algunas tendencias del derecho internacional a principios del siglo XXI", Anuario Mexicano de Derecho Internacional, vol. V, 2005.

Mazuelos Bellido, Ángeles, "Soft Law: ¿Mucho ruido y pocas nueces", Revista Electrónica de Estudios Internacionales, 2004, disponible en www.reei.org.

O'CONNELl, Mary Ellen, "The role of Soft Law in a Global Order", SHELton, Dinah (ed.), Commitment and compliance. The role of 
non-binding norms in the international legal system, New York, Oxford University Press, 2000.

PASTOR RIDRUeJO, José A., Curso de derecho internacional público y organizaciones internacionales, 8a. ed., Madrid, Tecnos, 2002.

REINICKE, Wolfgang, H. y MARTIN WitTE, Jan, "Interdependence, Globalization, and Sovereignty: The Role of Non-binding International Legal Accords", en SHelton, Dinah (ed.), Commitment and Compliance. The Role of Non-binding Norms in the International Legal System, New York, Oxford University Press, 2000.

REMIRO BROTÓNS, Antonio, "De los tratados a los acuerdos no normativos", en Ministerio de Asuntos Exteriores, La celebración de tratados internacionales por España: problemas actuales, Madrid, 1990.

_- RIQUelme, Rosa; ORIHUEla, Esperanza; DíEZ-HochleITNER, Javier y PÉREZ-PRAT, Luis, Derecho internacional, Madrid, McGraw-Hill, 1997.

RIEDEL, Eibe, "Standards and sources. Farewell to the exclusivity of sources triad in international law?", European Journal of International Law, 1991.

RIPHAGEN, W., "From soft law to ius cogens and back", Victorian University of Wellington Law Review, vol. 17, núm. 2, abril de 1987.

SÁNCHEZ RODRÍGUEZ, Luis Ignacio, "La apoteosis del consentimiento: de la noción de fuentes a los procesos de creación de derechos y de obligaciones internacionales", Anuario Hispano-Luso Americano de Derecho Internacional, vol. 16, 2003.

SEPÚlVEDA, César, "La diplomacia parlamentaria y la formación del nuevo orden jurídico internacional", en Estudios en Homenaje al profesor Miaja de la Muela, Madrid, Tecnos, 1979, t. II.

SHELTON, Dinah, "Law, Non-law and the problem of "Soft Law", en Commitment and compliance. The role of non-binding norms in the international legal system, New York, Oxford University Press, 2000.

SZTUCKI, Jerzy, "Reflections on International Soft law", en RAMBERG, Jan et al. (eds), Festskrift till Lars Hjerner. Studies in International Law, Stockholm, Norstedts, 1990.

TASIOULAS, John, "In Defence of Relative Normativity. Communitarian Values and the Nicaragua Case", Oxford Journal of Legal Studies, vol. 16, núm. 1. 
THÜRER Daniel, "Soft Law", en Bernhardt, R. (ed.), Encyclopedia of Public International Law, 2000.

TUNKIN, Grigory, "International law and political norms", en Hacia un nuevo orden internacional y europeo. Estudios en homenaje al profesor don Manuel Díez de Velasco, Madrid, Tecnos, 1993.

Virally, Michel, "Fuentes del derecho internacional", en Sorensen, Max (ed.), Manual de derecho internacional público, México, FCE, 1973.

WEIL, Prosper, “Towards Relative Normativity in Internacional Law?”, 77 American Journal of Internacional Law, 1983.

— - "Le Droit International en quête de son identité. Cours général de droit international public", Recueil des Cours 1992, The HagueBoston-London, VI, t. 237, 1996.

Wellens, K. C. y BorChardT, G. M., "Soft Law in European Community Law", European Law Review, October, 1989.

ZEMANEK, Karl, "Is the term 'Soft law' convenient?", en Liber Amicorum Professor Seidl-Hohenveldern, Kluwer, 1998.

- "The Legal Foundations of the International System. General Course on Public International Law", Recueil des Cours 1997, t. 266, 1998. 